\title{
Plans for a Neutron EDM Experiment at SNS
}

\author{
Takeyasu M. Itd 1 \\ Los Alamos National Laboratory, H846, PO Box 1663, Los Alamos, NM, 87545, USA \\ E-mail: ito@lanl.gov
}

\begin{abstract}
The electric dipole moment of the neutron, leptons, and atoms provide a unique window to Physics Beyond the Standard Model. We are currently developing a new neutron EDM experiment (the nEDM Experiment) 4]. This experiment, which will be run at the $8.9 \AA$ Neutron Line at the Fundamental Neutron Physics Beamline (FNPB) at the Spallation Neutron Source (SNS) at the Oak Ridge National Laboratory, will search for the neutron EDM with a sensitivity two orders of magnitude better than the present limit. In this paper, the motivation for the experiment, the experimental method, and the present status of the experiment are discussed.
\end{abstract}

\section{Introduction}

A nonzero permanent electric dipole moment (EDM) of a nondegenerate state of a system with spin $J \neq 0$ violates the invariance under time reversal as well as the invariance under parity operation. The violation of time reversal invariance implies a violation of invariance under $C P$ operation (combined operations of parity and charge conjugation) through the $C P T$ theorem.

Within the standard model (SM), in the electroweak sector $C P$ symmetry is broken by the complex phase $\left(\delta_{\mathrm{KM}}\right)$ in the CKM quark mixing matrix (the KM mechanism). To date, in laboratory measurements $C P$ violation has only been observed in $K$ and $B$ meson decays and the SM description of the $C P$ violation agrees with all the laboratory measurements to date. However, the question remains whether or not there are additional sources of $C P$ violation from new physics. Indeed, almost all extensions of the SM imply that there are such additional sources. Moreover, $C P$ violation is one of the necessary conditions for the matter-antimatter asymmetry observed in the Universe, and the SM and its description of $C P$ violation fail to accommodate the observed asymmetry. This discrepancy suggests that there are additional sources of $C P$ violation beyond that in the SM.

The current efforts to search for an electric dipole moment (EDM) of the neutron are motivated by the following two observations, which make the neutron EDM (as well as the EDM of other particles) an ideal place to search for new sources of $C P$ violation:

(i) In the KM mechanism, $C P$ violation only occurs in quark flavor changing processes to the lowest order. Therefore, the EDM due to this SM source of $C P$ violation is small; calculations predict it to be $\left|d_{n}\right| \sim 10^{-32}-10^{-31} e \cdot \mathrm{cm}$, several orders of magnitude smaller than the sensitivity possible with any experiment being considered at present.

(ii) Most extensions of the SM naturally produce larger EDMs because of additional $C P$ violating phases associated with additional particles introduced in the model.

1 For the nEDM Collaboration 
Furthermore, the SM has another source of $C P$ violation. This is a term in the QCD Lagrangian, the so-called $\theta$ term,

$$
\mathcal{L}_{Q C D}=\mathcal{L}_{Q C D, \theta=0}+\frac{\theta g_{s}^{2}}{32 \pi^{2}} G_{\mu \nu} \tilde{G}^{\mu \nu},
$$

which explicitly violates $C P$ symmetry because of the appearance of the product of the gluonic field operator $G$ and its dual $\tilde{G}$. Since $G$ couples to quarks but does not induce flavor change, $d_{n}$ is much more sensitive to $\theta$ than it is to $\delta_{\mathrm{KM}}$. Thus measurement of $d_{n}$ determines an important parameter of the SM. Calculations have shown that $d_{n} \sim \mathcal{O}\left(10^{-16} \theta\right) e \cdot \mathrm{cm}[1$. The observed limit on $d_{n}$ [2] provide a limit $\theta<\mathcal{O}\left(10^{-10}\right)$. A similar limit can be obtained from the observed limit on the EDM of the ${ }^{199} \mathrm{Hg}$ atom [3]. On the other hand, the natural scale suggests rather that $\theta \sim \mathcal{O}(1)$. The puzzle of why the value for $\theta$ is so small is called the strong $C P$ problem. Peccei and Quinn proposed a solution to this problem, in which the strong $C P$ is a conserved quantity. This solution, however, predicts the existence of a light pseudoscalar, called the axion. No axions have yet been observed despite extensive searches. It is important to establish experimentally if $\theta$ has a very small but finite value or $\theta$ is zero in order to shed light on this problem.

The current upper limit on the neutron EDM $d_{n}$ comes from a measurement performed at Institut Laue Langevin (ILL) [2] and is $\left|d_{n}\right|<2.9 \times 10^{-26} e \mathrm{~cm}(90 \%$ C.L.). With many theories predicting values lying within the six orders-of-magnitude window between the current limit and the value allowed by the SM, neutron EDM experiments that explore the next two orders of magnitude would make a significant contribution to the search for new physics that complements the information to be gained from the Large Hadron Collider (LHC).

We are currently developing a new neutron EDM experiment (the nEDM Experiment) [4]. This experiment, which will be run at the $8.9 \AA$ Neutron Line at the Fundamental Neutron Physics Beamline (FNPB) at the Spallation Neutron Source (SNS) at the Oak Ridge National Laboratory, will search for the neutron EDM with a sensitivity two orders of magnitude better than the present limit. A non-zero EDM will be a clear signal of physics beyond the SM, while a two-order-of-magnitude improvement on the limit will provide a significant challenge to many of the models of extensions of the SM.

\section{Experimental Method}

\subsection{Overview}

In the presence of a nonzero EDM, there is an interaction between the EDM and a static electric field that is analogous to the interaction between the magnetic moment and a static magnetic field. The Hamiltonian is

$$
H=-\left(\boldsymbol{\mu}_{n} \cdot \boldsymbol{B}+\boldsymbol{d}_{n} \cdot \boldsymbol{E}\right),
$$

where $\boldsymbol{B}$ and $\boldsymbol{E}$ are the applied static magnetic and electric fields, $\boldsymbol{\mu}_{n}$ is the magnetic moment, and $\boldsymbol{d}_{n}$ is the EDM of the neutron. The $\boldsymbol{d}_{n} \cdot \boldsymbol{E}$ interaction causes the neutron spin to precess when a neutron is placed in a static electric field, just like the $\boldsymbol{\mu}_{n} \cdot \boldsymbol{B}$ interaction does when the neutron is subject to a static magnetic field. Therefore, the EDM can be measured by looking for the change in spin precession frequency of the neutron associated with a reversal of $\boldsymbol{E}$ relative to $\boldsymbol{B}$. More specifically, the value of the EDM is given by

$$
d_{n}=\frac{h \Delta \nu}{4 E},
$$

where $h$ is the Planck constant, $\Delta \nu$ is the change in the precession frequency associated with a reversal of $\boldsymbol{E}$ relative to $\boldsymbol{B}$, and $E$ is the strength of the applied static electric field.

In a typical experimental arrangement, a sample of polarized neutrons is introduced into a volume where uniform magnetic and electric fields are applied, and the neutrons are let precess 
for a certain amount of time. In such experiments based on spin precession measurements, the statistical uncertainty $\left(\delta d_{n}\right)$ is ultimately given by the uncertainty principle and is 5 ]

$$
\delta d_{n}=\frac{\hbar}{2 E T \sqrt{m N}}
$$

where $T$ is the time for which the neutron spin is let precess, and $m$ represents the number of separate complete measurements of the $N$ neutrons.

For experiments in which stored ultracold neutrons (UCNs) 2 are used 3 where $N$ neutrons are stored for a time $T_{\text {store, }}$, in a given total time $t$ the number of measurements $m$ is inversely proportional to the storage time $T_{\text {store }}(m \sim t / T)$. Therefore,

$$
\delta d_{n} \propto \frac{1}{E \sqrt{N T_{\text {store }}}} .
$$

It is therefore obvious that in designing an EDM experiment, it is important to maximize $E$, $N$, and $T_{\text {store. }}$ In the most recent ILL experiment [2], $E=10 \mathrm{kV} / \mathrm{cm}, T_{\text {store }}=130 \mathrm{~s}$, and $N=1.4 \times 10^{4}$.

In the nEDM Experiment we intend to achieve a two orders of magnitude in sensitivity by adopting the method proposed by Golub and Lamoreaux [8], which is qualitatively different from the methods adopted in previous experiments (for the history of neutron EDM experiments, see for example Ref. [5]). The overall strategy can be summarized as follows:

- The experiment is performed in a bath of superfluid ${ }^{4} \mathrm{He}$.

- UCNs are produced locally in the measurement cells via the downscattering of $8.9 \AA$ cold neutrons in the superfluid helium (superthermal process).

- A dilute admixture of polarized ${ }^{3} \mathrm{He}$ atoms is introduced in the bath of superfluid ${ }^{4} \mathrm{He}$ and is used as the co-magnetometer.

- The polarized ${ }^{3} \mathrm{He}$ atoms are also used as the neutron spin analyzer; by observing the spindependent $n+{ }^{3} \mathrm{He} \rightarrow p+t$ reaction, the difference between the neutron and ${ }^{3} \mathrm{He}$ precession frequencies can be measured.

- The ${ }^{3} \mathrm{He}$ precession frequency is determined by directly measuring the change in the magnetic field caused by the rotating magnetization of the ${ }^{3} \mathrm{He}$ atoms using SQUID magnetometers.

With this strategy, we expect to achieve $E=50 \mathrm{kV} / \mathrm{cm}, T_{\text {store }} \sim 500 \mathrm{~s}$, and $N \sim 10^{6}$, resulting in a two-orders-of-magnitude improvement in sensitivity. Some of the important features of this strategy will be described in more details below.

\subsection{UCN Production}

In previous EDM experiments with stored UCNs, UCNs produced elsewhere were transported to the experimental apparatus and were stored in the measurement cells. The number of UCNs suffer from the loss in the extraction of UCNs from the UCN source and during the transport from the source to the experiment. These problems can be overcome if UCNs can be generated directly in the EDM measurement cell. It is in fact possible using the superthermal process [9]

2 Ultracold neutrons are neutrons with total kinetic energy less than the effective potential $U_{F}$ presented by a material boundary. These neutrons, therefore, can be confined in a material bottle. Typically $U_{F} \sim 200 \mathrm{neV}$, which corresponds to velocities of order $5 \mathrm{~m} / \mathrm{s}$, wavelengths of order $500 \AA$ and an effective temperature of order $2 \mathrm{mK}$. See, for example Ref. 6]

3 The use of UCNs is important in suppressing the motional magnetic field effects 7, which were a major limitation in early experiments with a cold neutron beam. 


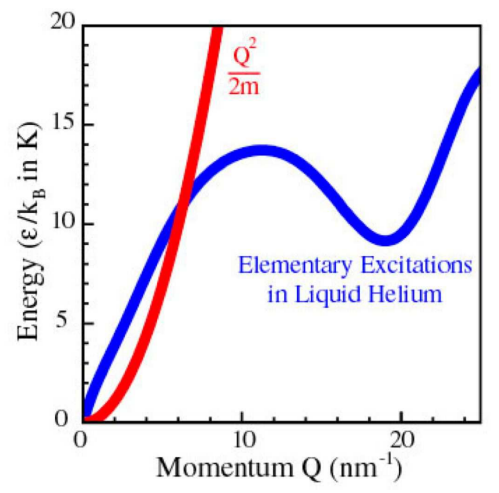

Figure 1. The dispersions curve for the elementary excitation in superfluid ${ }^{4} \mathrm{He}$ (blue) and the dispersion curve for the free neutron (red). The two curves cross at $Q=0$ and $Q=Q^{*}$, which corresponds to a neutron wavelength of $8.9 \AA$ or an energy of $12 \mathrm{~K}$.

in superfluid liquid ${ }^{4} \mathrm{He}$ with 8.9 A neutrons delivered to the measurement cell. This mechanism is illustrated in Fig. 1. Shown in the figure are the dispersion curve of the elementary excitation in superfluid ${ }^{4} \mathrm{He}$ and that of the free neutron, which cross at $Q=0$ and $Q=Q^{*}$, which corresponds to a neutron wavelength of $8.9 \AA$ or an energy of $12 \mathrm{~K}$. Therefore, neutrons with a wavelength of $8.9 \AA$ can give all their energy and momentum to the elementary excitations in superfluid helium and become ultracold neutrons. This process is called "downscattering." The reverse process "upscattering" is highly suppressed, because there are hardly any elementary excitations with energy of $E^{*}=12 \mathrm{~K}\left(Q=Q^{*}\right)$ when the temperature of the liquid helium is $T<0.5 \mathrm{~K}$ (suppressed by $\left.\exp \left(-E^{*} / k T\right)\right)$. The production of UCNs using this method has been experimentally demonstrated [10, 11].

Since ${ }^{4} \mathrm{He}$ has zero neutron absorption, UCN can be store in the bath of superfluid ${ }^{4} \mathrm{He}$ until $\beta$ decay, wall absorption, or upscattering occurs. Our goal is to achieve a $500 \mathrm{~s}$ lifetime for the stored UCNs. With this and the expected neutron flux at the $8.9 \AA$ Line at FNPB (see Sec (3)), we expect to have $N \sim 10^{6}$ per measurement. We plan to use a beam of polarized neutrons to generate UCNs polarized.

There are other advantages to performing an EDM experiment directly in a bath of liquid helium. A dilute admixture of polarized ${ }^{3} \mathrm{He}$ atoms can be introduced into the bath, and can serve as a comagnetometer and neutron spin analyzer as we will see below. In addition, because of the excellent dielectric properties of liquid helium, it is expected that a higher electric field can be applied in liquid helium than in vacuum.

\subsection{Magnetic Field Measurement with $a^{3}$ He Comagnetometer}

An EDM of $10^{-28} e \mathrm{~cm}$ would produce a relative change of $\sim 5 \times 10^{-9} \mathrm{~Hz}$ in precession frequency on reversal of $\boldsymbol{E}$ with respect to $\boldsymbol{B}$ for $|\boldsymbol{E}|=50 \mathrm{kV} / \mathrm{cm}$. This frequency shift corresponds to a change in magnetic field of about $2 \times 10^{-12}$ gauss. Obviously extreme care needs to be taken to ensure and monitor the temporal stability and spatial uniformity of the magnetic field in order 
to minimize any possible systematic effects.

We will use polarized ${ }^{3} \mathrm{He}$ atoms as comagnetometer as a means to address this issue.. A comagnetometer is a polarized atomic species within the same storage volume as the neutrons, which provides a nearly exact spatial and temporal average of the magnetic field affecting the neutrons over the storage period. The effectiveness and importance of the use of comagnetometer have recently been demonstrated experimentally in the ILL experiment [2, 12].

We will introduce a dilute admixture of polarized ${ }^{3} \mathrm{He}$ atoms into the superfluid ${ }^{4} \mathrm{He}$. The fractional density of ${ }^{3} \mathrm{He}$ will be $x=N\left({ }^{3} \mathrm{He}\right) / N\left({ }^{4} \mathrm{He}\right) \sim 10^{-10}$ (This is determined from the neutron lifetime in the helium bath, as discussed below). ${ }^{3} \mathrm{He}$ is a diamagnetic atom and the EDM of ${ }^{3} \mathrm{He}$ is negligible due to the shielding from the two bound electrons. The precession of ${ }^{3} \mathrm{He}$ atoms will be determined by directly measuring the change in the magnetic field caused by the rotating magnetization of the ${ }^{3} \mathrm{He}$ atoms using SQUID magnetometers.

\subsection{Measurement of the Neutron Precession Frequency}

The ${ }^{3} \mathrm{He}$ atoms also serves as an analyzer of the neutron precession frequency. The cross section for neutron absorption by ${ }^{3} \mathrm{He}$ is strongly spin dependent: $59 \mathrm{~b}$ for spins aligned and $11 \mathrm{~kb}$ for spins opposite at thermal neutron energy $(25.3 \mathrm{meV})$. The cross sections for both scale inversely with neutron velocity. Thus if the ${ }^{3} \mathrm{He}$ concentration is adjusted to $10^{12}$ atoms/cc $\left(x=N\left({ }^{3} \mathrm{He}\right) / N\left({ }^{4} \mathrm{He}\right) \sim 10^{-10}\right)$, then the neutrons are only absorbed when the neutron spin is opposite to the ${ }^{3} \mathrm{He}$ spin. Therefore the rate of neutron absorption on ${ }^{3} \mathrm{He}$ is proportional to

$$
1-P_{3} P_{n} \cos \left[\left(\gamma_{3}-\gamma_{n}\right) B_{0} t\right],
$$

where $P_{3}$ and $P_{n}$ are the polarizations of the ${ }^{3} \mathrm{He}$ and the neutrons respectively, and $\gamma_{3}$ and $\gamma_{n}$ are the gyromagnetic ratios of the ${ }^{3} \mathrm{He}$ and the neutrons respectively.

When a neutron is absorbed on a ${ }^{3} \mathrm{He}$ atom, the reaction products are a proton and a triton that share $764 \mathrm{keV}$ of energy. When the reaction products travel in liquid helium, they produce scintillation light in the hard ultraviolet $(\sim 80 \mathrm{~nm})$. If the measurement cells are coated with a wavelength shifter, the scintillation light is converted to the blue which may be detected by photomultiplier tubes (PMT).

By measuring the rate of scintillation light, the difference between the neutron precession frequency and the ${ }^{3} \mathrm{He}$ precession frequency can be determined. Note that the ${ }^{3} \mathrm{He}$ precession frequency is measured using the SQUID magnetometer. The signature of a neutron EDM would appear as shift in the neutron $-{ }^{3}$ He precession frequency difference corresponding to the reversal of the $\boldsymbol{E}$ relative to $\boldsymbol{B}$ with no corresponding change in ${ }^{3} \mathrm{He}$ precession.

\subsection{Dressed Spin Technique}

In addition to the use of SQUID magnetometer to monitor the polarized ${ }^{3} \mathrm{He}$, the so-called "dressed-spin technique" can be used to look for the signature of the neutron EDM. This is based on the fact that in the presense of a strong oscillating magnetic field, the magnetic moment of a particle is modified or "dressed", yielding an effective gyromagnetic ratio given by

$$
\gamma^{\prime}=\gamma J_{0}\left(\gamma B_{R F} / \omega_{R F}\right)=\gamma J_{0}(\gamma x)
$$

where $\gamma$ is the unperturbed gyromagnetic ratio, $B_{R F}$ and $\omega_{R F}$ are the amplitude and frequency of the applied oscillating magnetic RF field, $x=\gamma B_{R F} / \omega_{R F}$, and $J_{0}$ is the zero-th order Bessel function. Thus by applying an RF field perpendicular to the static $B_{0}$ field that satisfies

$$
\gamma_{n} J_{0}\left(\gamma_{n} x\right)=\gamma_{3} J_{0}\left(\gamma_{3} x\right)
$$

the neutrons and ${ }^{3} \mathrm{He}$ atoms can be made to precess at the same frequency (the subscripts $n$ and 3 refer to the neutrons and the ${ }^{3} \mathrm{He}$ atoms respectively). This condition is called 


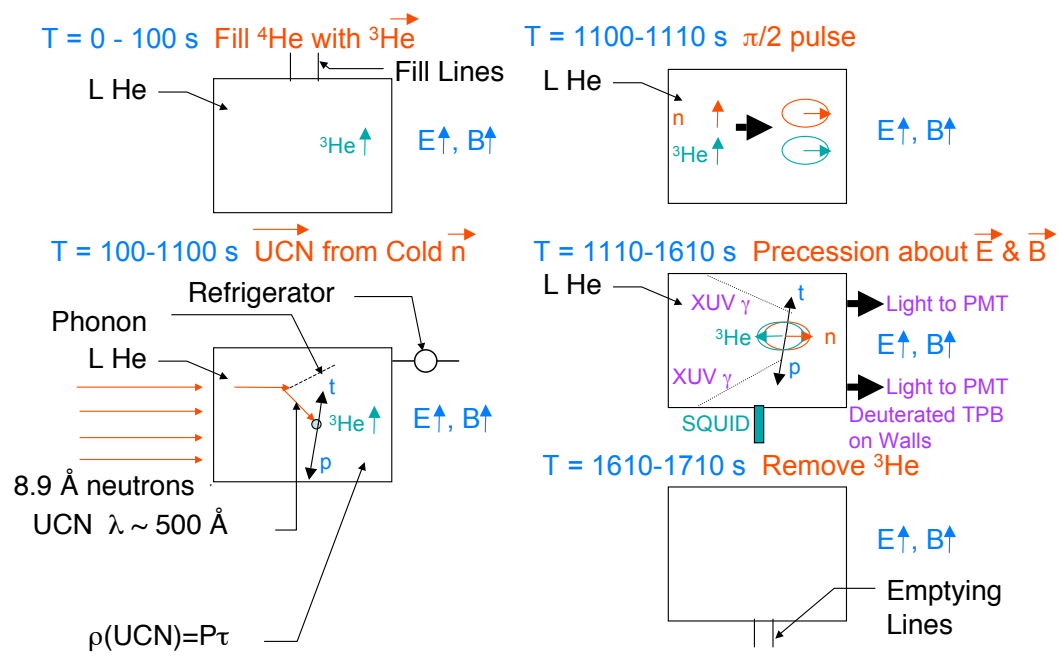

Figure 2. Illustrative description of a proposed measurement cycle. The duration of each step remains to be optimized to achieve maximal sensitivity once the UCN storage and ${ }^{3} \mathrm{He}$ relaxation times in the measurement cell is known.

"critical dressing." In practice, one adjusts the dressing RF field parameter $x$ by eliminating the oscillating component of the scintillation light. The signature of a neutron EDM would appear as the change in $x$ for critical dressing corresponding to the reversal of the $\boldsymbol{E}$ relative to $\boldsymbol{B}$. An EDM experiment insensitive to background magnetic field can thus be performed.

\subsection{Measurement Cycle}

To summarize the method described above, a proposed measurement cycle is illustrated in Fig. 2. Note that the duration of each step remains to be optimized to achieve maximal sensitivity once the UCN storage and ${ }^{3} \mathrm{He}$ relaxation times in the measurement cell are known.

\section{FNPB at SNS}

The experiment will be performed at the Fundamental Neutron Physics Beamline (FNPB) at the Spallation Neutron Source (SNS) at the Oak Ridge National Laboratory. The Spallation Neutron Source (SNS), currently under construction at the Oak Ridge National Laboratory, is an accelerator-based neutron source, and will provide the world's most intense pulsed neutron beams for scientific research and industrial development [13. The Fundamental Neutron Physics Beamline (FNPB), one of the 24 neutron beamlines in the SNS target hall, is dedicated to fundamental physics using cold and ultracold neutrons.

The FNPB has two neutron beamlines, the "Cold Neutron Line" and the 8.9 A Line (or "UCN Line"). The $8.9 \AA$ Line is dedicated to experiments that will uses the superthermal process in superfluid liquid helium to produce ultra-cold neutrons. The $8.9 \AA$ neutrons will be selected by a double crystal monochromator and will be sent to an external building located about $30 \mathrm{~m}$ downstream, where the nEDM Experiment will be mounted.

The construction of SNS completed in 2006. Currently the beam power is being ramped up towards its full-power capacity of 1.4 MW. The FNPB is currently under construction. The construction is planned to be completed in early 2010 .

\section{Experimental Apparatus}

The conceptual design of the proposed apparatus is shown in Fig. 3. The picture is derived from a full 3-D engineering model that has been created to study whether all the scientific ideas can be 


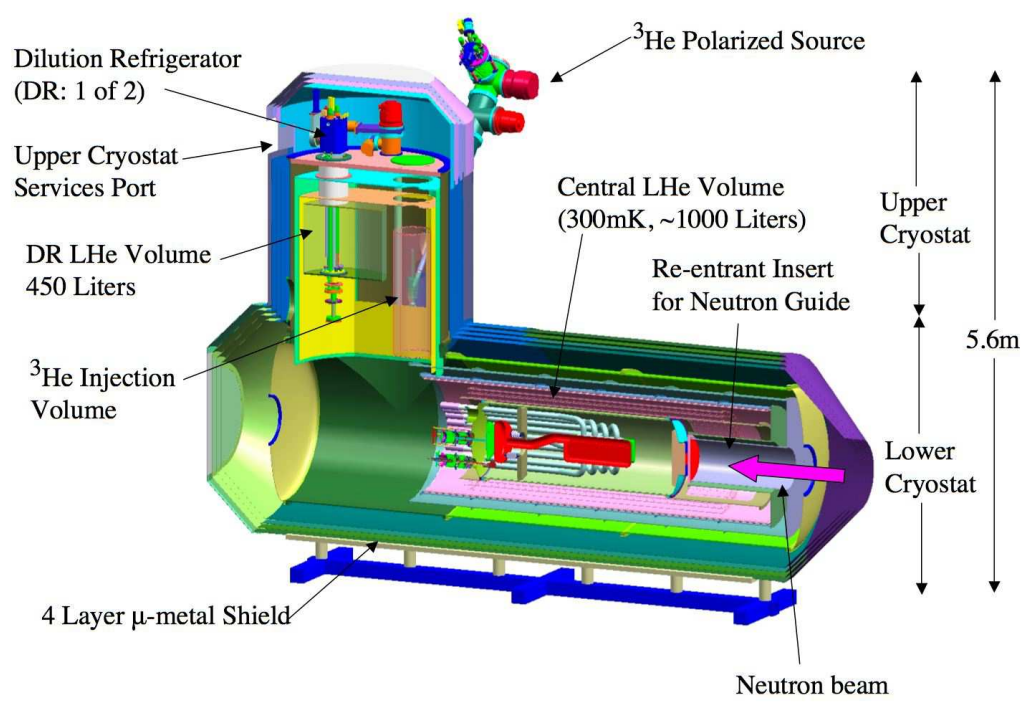

Figure 3. The schematic overview of the full detector apparatus.

realized in single piece of equipment. The apparatus is divided into two parts, the lower cryostat where the measurement is made and the upper cryostat where the ${ }^{3} \mathrm{He}$ is injected and removed as well as where the refrigeration is done. Both lower and upper cryostats are surrounded by four layers of $\mu$-metal magnetic shields to shield the apparatus from the ambient magnetic field and its temporal change. Two neutron guides (not shown), which provide polarized $8.9 \AA$ neutrons, enter from the right and terminate roughly $50 \mathrm{~cm}$ upstream of the high voltage plates.

The cutaway view of the lower cryostat is shown in Fig. 4, There are two measurement cells, which are placed in the gaps between the high voltage and ground electrodes. The cells are made of acrylic, and the inner walls are coated with deuterated polystyrene to minimize neutron absorption by hydrogen. The deuterated polystyrene is loaded with deuterated tetraphenyl butadinene (dTPB) which serve as the wavelength shifter for the hard ultraviolet scintillation light from the neutron absorption on ${ }^{3} \mathrm{He}$. The converted light is guided through the light guides and is detected by PMTs.

The high voltage of about $350 \mathrm{kV}$ is necessary to generate the $50 \mathrm{kV} / \mathrm{cm}$ electric field across the $7 \mathrm{~cm}$ wide measurement cells. A novel technique to supply $\mathrm{HV}$ without need for direct $350 \mathrm{kV}$ application is being developed but we are also developing a high voltage feedthrough for direct application of the necessary voltage.

The measurement cells, the light guides, the electrodes, and the variable capacitor are all immersed in a 1200-liter bath of superfluid helium which serve as the insulator for the high voltage system. Also the superfluid helium bath surrounding the cells help keep the temperature across the cells uniform, and eliminate potential heat sources. This is important because a heat source can generate a phonon wind which blows away ${ }^{3} \mathrm{He}$ atoms thus generating a nonuniformity in ${ }^{3}$ He concentration. The SQUID magnetometers are mounted on the ground plates.

A superconducting magnetic shield and a ferromagnetic shield provide a magnetic shielding in addition to the conventional ( $\mu$-metal) room temperature shields. Located between the 


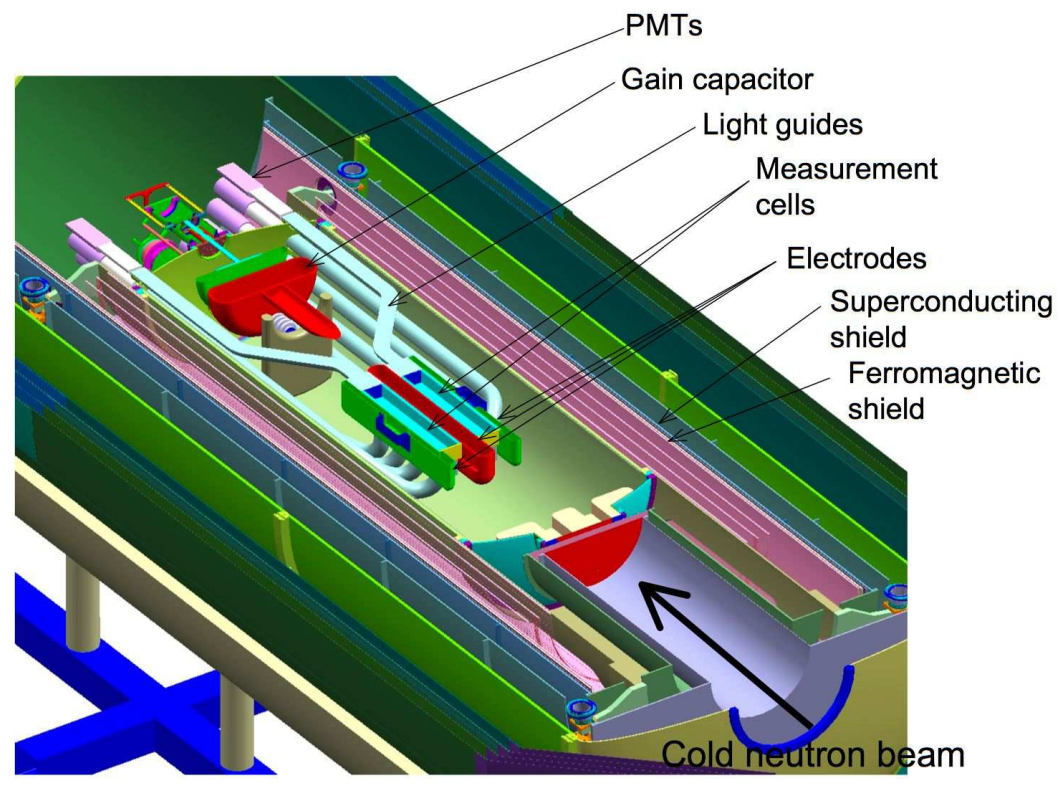

Figure 4. The central region of the detector illustrating the measurement cells, electrodes, light guides, HV generator and magnetic shields.

ferromagnetic shield and the 1200-liter insulating helium volume are various coils that generate a uniform static $10 \mathrm{mG}$ field ( $B_{0}$ field), RF pulses to rotate the spins of the neutrons and ${ }^{3} \mathrm{He}$ $(\pi / 2$ pulse), and RF pulses for the dressing field.

\section{Status and Plans}

Currently, the collaboration is refining the conceptual design as well as vigorously pursuing various $R \& D$ studies $[15-25]$ that are necessary to optimize the final design of the experiment. The ongoing and complete R\&D topics include (but are not limited to):

- study of the neutron storage time in an acrylic cell coated with deuterated polystyrene

- study of dielectric properties of superfluid helium [15]

- theoretical and experimental study of the possible systematic effects due to the interference between the motional magnetic field effects and the gradient of the $B_{0}$ field [16, 17]

- modeling and prototyping of various coils

- study of the neutron beam line [18]

The R\&D and the design will continue through calendar year 2007. The construction is expected to start subsequently. The operation of the apparatus is expected to start around 2013.

\section{References}

[1] M. Pospelov and A. Ritz, Phys. Rev. Lett. 83, 2526 (1999).

[2] C. A. Baker et al., Phys. Rev. Lett. 97, 131801 (2006).

[3] M. V. Romalis, W. C. Crifith, J. P. Jacobs, and E. N. Fortson, Phys. Rev. Lett. 86, 2505 (2001). 
[4] The nEDM Experiment (M. D. Cooper and S. K. Lamoreaux spokespersons), http:p25ext.lanl.gov/edm/edm.html.

[5] I. B. Khriplovich and S. K. Lamoreaux, CP Violation Without Strangeness (Springer-Verlag, Berlin 1997).

[6] R. Golub, D. Richardson, and S. K. Lamoreaux, Ultra-Cold Neutrons (Adam Hilger, Bristol, 1991).

[7] P. G. H. Sanders and E. Lipworth, Phys. Rev. Lett. 13, 718 (1964).

[8] R. Golub and S. K. Lamoreaux, Phys. Rep. 237, 1 (1994)

[9] R. Golub and J. M. Pendlebury, Phys. Lett. A 62, 337 (1977).

[10] P. Ageron et al., Phys. Lett. A 66, 469 (1978).

[11] R. Golub et al., Z. Phys. B 51, 187 (1983).

[12] P. G. Harris, et al., Phys. Rev. Lett. 82, 904 (1999).

[13] The Spallation Neutron Source, http://www.sns.gov/

[14] Fundamental Neutron Physics Beamline, http://www.phy.ornl.gov/nuclear/neutrons/

[15] J. C. Long, et al., physics/0603231.

[16] S. K. Lamoreaux and R. Golub, Phys. Rev. A 71, 032104 (2005).

[17] A. L. Barabanov, R. Golub, and S. K. Lamoreaux, Phys. Rev. A 74, 052115 (2006).

[18] T. M. Ito, C. B. Crawford, and G. L. Greene, Nucl. Instrum. Methods Phys. Res. Sect A 564, 414 (2006).

[19] A. O. Sushkov, et al., Phys. Rev. Lett. 93, 153003 (2004).

[20] M. E. Hayden, S. K. Lamoreaux, and R. Golub, AIP Conf. Proc. 850, 147 (2006).

[21] G. Archibald, et al., AIP Conf. Proc. 850, 143 (2006).

[22] C. P. Bidinosti, I. S. Kravchuk, and M. E. Hayden, J. Magn, Reson 177, 31 (2005).

[23] M. E. Hayden, et al., Phys. Rev. Lett. 93, 105302 (2004).

[24] M. E. Hayden, et al., Physica B 329, 236 (2003).

[25] S. K. Lamoreaux, et al., Europhys. Lett. 58, 718 (2002). 